\title{
The Steroid-Inducible pOp6/LhGR Gene Expression System is Fast, Sensitive and Does NOT Cause Plant Growth Defects in Rice (Oryza Sativa)
}

Marketa Samalova ( $\sim$ marketa.samalova@sci.muni.cz)

Masaryk University

lan Moore

Oxford University

\section{Research Article}

Keywords: Dexamethasone, glucocorticoid, chemically inducible gene expression, GVG, XVE, promotor, monocots

Posted Date: June 24th, 2021

DOI: https://doi.org/10.21203/rs.3.rs-580542/v1

License: (c) (i) This work is licensed under a Creative Commons Attribution 4.0 International License.

Read Full License 


\section{Abstract}

Inducible systems for transgene expression activated by a chemical inducer or an inducer of non-plant origin are desirable tools for both basic plant research and biotechnology. Although, the technology has been widely exploited in model plants, it has not been optimised for use with the major monocotyledonous crop species, namely rice. We have adapted the dexamethasone-inducible p0p6/LhGR system for rice and shown that it is fast, sensitive and tightly regulated, with high levels of induction that remain stable over several generations. Most importantly, we have shown that the system does not cause negative growth defects in vitro or in soil grown plants. Interestingly in the process of testing, we found that another steroid, triamcinolone acetonide, is a more potent inducer in rice than dexamethasone. We present serious considerations for the construct design to avoid undesirable effects caused by the system in plants, leakiness and possible silencing, as well as simple steps how to maximize translation efficiency of a gene of interest. Finally, we compare the performance of the p0p6/LhGR system with other chemically inducible systems tested in rice in terms of the properties of an ideal inducible system.

\section{Background}

Chemically inducible systems that regulate gene expression are crucial tools for basic plant biology research as well as biotechnology applications. They allow analysis of gene primary effects before homeostatic mechanisms start to counteract and reveal a clear correlation between induction of a transgene and occurrence of an altered phenotype. Their applications include expression of gene products that interfere with regeneration, growth or reproduction; regulation and expression at different stages of plant development; conditional genetic complementation, co-suppression and overexpression studies. However, adaption of existing inducible systems developed in model plants to other species including monocots and important crop plants particularly rice, has not been easily achieved.

The systems typically contain two transcription units. The first unit employs a constitutive or tissuespecific promoter to express a chemical-responsive transcription factor, the second unit consists of multiple copies of the transcription factor binding site linked to a minimal plant promoter, which is used to express the target gene. The most optimal systems developed to date include dexamethasoneinducible GVG (Aoyama and Chua, 1997) and pOp6/LhGR (Craft et al., 2005; Samalova et al., 2005), estrogen-inducible XVE (Zuo et al., 2000), ecdysone agonist-inducible VGE (Padidam et al., 2003; Koo et al., 2004) and ethanol-inducible alc systems (Caddick et al., 1998; Roslan et al., 2001; Salter et al., 1998). Their characteristics were compared in detail by Moore et al., (2006) and potential applications in plant biotechnology were reviewed by Corrado and Karali, (2009).

Several attempts have been made to develop an inducible system for gene manipulation in rice. The XVE system was tested by Sreekala et al., (2005) and Okuzaki et al., (2011) but the use remains very limited due to the lack of systemic movement of the estradiol inducer (Hirose et al., 2012, Chen et al., 2017). Two other steroid-inducible systems have been tested but the GVG and a modular gene expression system 
(Vlad et al., 2019) derived from the p0p6/LhGR system met with a mixed success in rice, as they caused severe growth and developmental defects of the plants (Ouwerkerk et al., 2001; Vlad et al., 2019).

An easier and more common strategy exploited in rice is the use of conditional promotors that can be activated by heat (Khattri et al., 2011; Sun et al., 2012; Rerksiri et al., 2013), pathogens (Helliwell et al., 2013; Quilis et al., 2014) or wounding (Quilis et al., 2014), oxidative (Woo et al., 2015) and other stresses (Nakashirma et al., 2007). Other crops promoters include induction by heat in maize (Du et al., 2019) and potato (Kopertekh et al., 2018), by light in tomato (Timerbaev and Dolgov, 2019) or, cold in barely (Eva et al., 2018), wheat (Meszaros et al., 2015) and sweet potato (Honma et al., 2019). Often the conditional expression is combined with the Cre-lox technology (Khattri et al., 2011; Meszaros et al., 2015; Eva et al., 2018; Kopertekh et al., 2018; Du et al., 2019) or an alternative site-specific recombinase system (Woo et al., 2015) to generate marker-free genetically modified plants.

Adaptation of the breakthrough CRISPR/Cas9 technology to plants (Jiang et al., 2013) including rice (Zhang et al., 2014) allows genome editing and creates a powerful tool for engineering knockdown, knockout or chimeric plants. This genome editing system was combined with a heat-shock-inducible promoter to generate heritable mutations in rice (Nandy et al., 2019) and a virus-inducible system was developed that confers resistance to Gemini viruses in model plants Arabidopsis and tobacco (Ji et al., 2018). Recently, the technology was integrated with the estradiol-inducible XVE-based cell-type-specific system (Siligato et al., 2016; Zuo et al., 2000) to create an inducible genome editing (IGE) system in Arabidopsis that enables efficient generation of target gene knockouts in desired cell types at different developmental stages (Wang et al., 2020).

Over the years we and others have made considerable efforts to develop the dexamethasone-inducible transcription activation system, p0p6/LhGR, as a tool for the growing demands on modern gene technologies. It is a widely used system for which a comprehensive library of cell-type specific activator lines was created in Arabidopsis (Schurholz et al., 2018); the system was combined with artificial microRNA (amiRNA) to knockdown multigene expression (Goth et al., 2012; Samalova et al., 2020) and hairpin RNAi molecules to silence gene expression (Wielopolska et al., 2005; Liu and Yoder, 2016). Apart from Arabidopis (Craft et al., 2005) and tobacco (Samalova et al., 2005) the system was tested in various other species including citrus plant (Rossignol et al., 2014) and Medicago truncatula (Liu and Yoder, 2016) and most recently in rice (Vlad et al., 2019). However, the specific modifications made to the original version of the pOp6/LhGR system caused undesirable effects in plants.

This report describes the functionality of the p0p6/LhGR system in rice, its stability over several generations, time course and dose response characteristics; optimization of induction by various steroids as inducers and methods of systemic and localised applications that do not have any detrimental effects in rice even after prolonged induction.

\section{Results}


The p0p6/LhGR system (Craft et al., 2005; Samalova et al., 2005) comprises of a transcription activator LhGR which is a fusion between a high-affinity DNA-binding mutant of Escherichia coli lac repressor, lact ${ }^{\text {His17 }}$, transcription-activation-domain-II of Gal4 from Saccharomyces cerevisiae and the ligandbinding domain (LBD) of the rat glucocorticoid receptor (GR). The p0p6 is a chimeric promoter that consists of six copies of lac operators ( $/ a c O p$ ) cloned upstream of a minimal cauliflower mosaic virus (CaMV) 35S promoter $(-50$ to +8$)$ and is apparently silent when introduced into plants. The principle of the system is that in the absence of the steroid ligand, dexamethasone (Dex), the transcription factor is trapped in an inactive complex via interaction of the GR LBD and heat-shock protein HSP90. Upon induction with Dex, this complex is disrupted and the LhGR activator binds to the p0p6 promotor and induces expression of the target gene of interest.

To adopt the p0p6/LhGR system for rice, first we chose a binary pVec8-overexpression vector (Kim and Dolan, 2016) in which we placed the LhGR2 activator sequence that incorporates the Arabidopsis codonoptimized GAL4 sequence (Rutherford et al., 2005) under the control of a maize ubiquitin promoter that contains an intron $(p Z m U b i)$. The inclusion of an intron is well known to greatly increase expression efficiency in monocots, but similar effects have been reported in dicots and other eukaryotes (Rose, 2004). Secondly, we checked in the literature (Mitsuhara et al., 1996; Segal et al., 2003) that none of the sequences of the pOpln2 bidirectional reporter cassette (Samalova et al., 2019) including the lacOp, minimal promoters and tobacco mosaic virus (TMV) omega $(\Omega)$ translation enhancers have been reported to be toxic or non-functional in monocots and cloned it into the activator construct to create pVecLhGR2 as depicted in Fig. 1 and Supp. Fig. 1. For simplicity of testing the regulated expression of the pOp6/LhGR in rice, we used the uidA (encoding b-glucuronidase; GUS) and the yellow fluorescent protein $(Y F P)$ as the genes of interest.

To create stable transgenic rice lines, we used a protocol for Agrobacterium-mediated transformation of calli induced from seeds of Oryza sativa spp. japonica cultivar Kitaake as described by Vlad et al., 2019. We generated several independent transgenic lines (T1) in which the induced GUS staining was comparable to that from the constitutive promoter $p Z m U b i$ (Fig. 1B) and the YFP expression (Fig. 1C) was inducible by Dex, proving that the p0p6/LhGR system is in principle functional in rice. However, the transformation efficiency was relatively low and to induce higher levels of expression various alternate methods were tested.

\section{Transformation efficiency and reliability of induction in subsequent generations}

We tested GUS activity by histochemical staining in 120 generated putative transformants that included little plantlets with a piece of callus and some roots (Table 1). We induced them in liquid $1 / 2 \mathrm{MS}$ with $30 \mathrm{mM}$ Dex and stained for GUS activity 24h later. Approximately a half of them showed visible (by eye) GUS staining in roots after $2 \mathrm{~h}$ and shoots after $4 \mathrm{~h}$. The reaction was stopped and scored at $24 \mathrm{~h}$ with the following pattern: $30.8 \%$ root staining only, $9.2 \%$ shoots only, $12.5 \%$ stained shoots, roots and callus (data not shown). 
One hundred and sixty-six putative primary transformants (T0) were grown to maturity and tested. The first generation of seedlings (T1) were tested for resistance to hygromycin by germinating them on $1 / 2$ MS medium supplemented with the antibiotics (Table 1). Only 33 lines germinated and grew, indicating that only $20 \%$ were real transformants, of these, 8 lines (25\%) showed positive GUS staining after induction with Dex. Five of the most strongly inducible lines were grown to the next generation (T2), these lines retained HYG-resistance and showed positive GUS staining upon Dex induction. Two lines (65 and 121) were tested further and showed stable inducible GUS expression in the subsequent (T3) generation (Fig. 2A).

We determined the GUS activity fluorometrically in segregating T1 and T2 progeny in roots (Fig. 2B) and shoots (Fig. 2C) of 7-day-old rice seedlings germinated and grown on $1 / 2$ MS plates containing $30 \mathrm{mM}$ Dex or the same concentration of DMSO (-Dex control). Interestingly, the induced GUS activity was up to 8-fold higher in roots compared to shoots and in some cases in roots it was comparable to the activity from the constitutive pZmUbi promoter. Perhaps a low transpiration rate in Petri dishes impaired the uptake and distribution of Dex into the shoots.

\section{Time course and dose response characteristics of Dex-induced GUS activity}

To characterise the induction property of the p0p6/LhGR system in rice we performed time course and dose response experiments. To increase the efficiency of induction in shoots, we induced the newly developed leaves of app. 2-week old seedlings, grown in the open air, by painting the leaves with a Dex solution supplemented with $0.1 \%$ (v/v) Tween-20. Significant increase in fluorometrically, determined GUS activity, was detected $12 \mathrm{~h}$ after induction with $10 \mathrm{mM}$ Dex in two independent transgenic lines (65B and $121 \mathrm{C}$ ) and this activity reached app. a half of the $p Z m U b i$ constitutive promoter activity within $72 \mathrm{~h}$ of induction (Fig. 3A). The GUS activity was induced in plants treated with $0.01 \mathrm{mM}$ Dex and while one line (65B) reached maximum levels of induction with $0.1 \mathrm{mM}$ Dex, the other line (121C) had increased activity with increasing Dex concentration and reached levels similar to the constitutive pZmUbi promoter with $10 \mathrm{mM}$ Dex after 48h induction (Fig. 3B).

To confirm the similar characteristic of the p0p6/LhGR system in roots, we induced detached roots of 10day old seedlings in liquid $1 / 2$ MS media supplemented with increasing concentrations of Dex and performed histochemical GUS staining after specific time durations. Visible GUS staining was first detected in developing lateral roots and tips after $12 \mathrm{~h}$ of induction and the intensity increased throughout the root system up to $72 \mathrm{~h}$ tested (Fig. $3 \mathrm{C}$ ). The maximal GUS staining intensity was detected with $1 \mathrm{mM}$ Dex, the lowest concentration tested (Fig. 3D), predominantly in growing root tips after $24 \mathrm{~h}$ induction.

We also tested the feasibility of inducing whole seedlings (10-day old) in a liquid $1 / 2$ MS medium supplemented with $10 \mathrm{mM}$ Dex. Histochemical GUS staining revealed the induction after $12 \mathrm{~h}$ predominantly in roots and the staining pattern did not change significantly in the $48 \mathrm{~h}$ time-span tested (data not shown).

\section{Optimization of induction by testing different steroids as inducers}


We tried to improve the levels of induction of the p0p6/LhGR system in rice by testing different glucocorticoid derivatives (steroids) as inducers. In an attempt to reduce the surface tension at the airliquid interface that is high in rice leaves due to epicuticular waxes preventing water vapor loss, we tested different concentrations of Tween-20 as the wetting agent rather than Silwet L-77 used previously (Craft et al., 2005; Samalova et al., 2005). Fig. 4A (i-iv) shows clear differences in the intensity of GUS staining of the 10-day-old shoots (leaves and stems) induced with a $30 \mathrm{mM}$ Dex solution supplemented with $0.1 \%$ Tween-20 compared to $0.01 \%$. Almost no staining was visible without the addition of the surfactant apart from damaged cells.

It is known that other glucocorticoid derivatives such as triamcinolone acetonide (TA) or deoxycorticosterone (Doc) can be used as inducers to replace Dex (Aoyama and Chua, 1997). We tested both in a $30 \mathrm{mM}$ water solution supplemented with $0.1 \%$ Tween-20 (Fig. 4A v and vi) and compared the GUS staining intensities. Doc induction was negligible; however, TA induction was comparable if not higher than with Dex. To confirm this observation, we repeated the experiment on cuttings of the same leaf of 6-week-old plants that were submerged in water supplemented with $0.1 \%$ Tween-20 and $10 \mathrm{mM}$ Dex or $10 \mathrm{mM}$ TA for 24h (Fig. 4B). Depending on the efficiency of the substrate penetration, the GUS staining pattern with both inducers was comparable, but more importantly, also comparable to the staining of pZmUbi::GUS line of the same age. No GUS staining was detected without the inducers (Fig. 4B, $0.1 \%$ Tween-20).

We expanded the range of possible steroid inducers readily available and tested them in a $30 \mathrm{mM}$ water solution supplemented with $0.1 \%$ Tween-20. Leaves of 5-week-old plants (Fig. 4C, lines 65A top and $121 \mathrm{C}$ bottom) were painted with the following steroids: betamethasone (Bet), fludrocortisone acetate (Flu), prednisone (Pre) or prednisolone (Plo) and Dex and histochemical GUS staining was carried out 24h later. None of the new glucocorticoids reached GUS activity levels comparable to Dex in both the transgenic lines tested and the GUS staining was considerably weaker than that of the constitutive $p Z m U b i$ promotor activity.

\section{Systemic and localised induction of soil-grown plants}

To test the feasibility of inducing gene expression at later stages of plant development, either in a systemic or in a localized manner, Dex or the glucocorticoid TA were applied to soil-grown plants by watering (subterranean irrigation) or painting. Local induction of expression was detected after $24 \mathrm{~h}$ when leaves were painted with a $10 \mathrm{mM}$ steroid solution supplemented with $0.1 \%$ Tween-20 (Fig. 5A). The treatment was repeated and GUS activity determined $24 \mathrm{~h}$ and $72 \mathrm{~h}$ following first application of the inducer. Interestingly, the TA treatment doubled the induced levels of GUS activity compared to Dex, and both treatments exceeded the levels of $p Z m U b i$ promoter activity at $72 \mathrm{~h}$ post induction (hpi).

Similarly, plants were watered with a $30 \mathrm{mM}$ steroid solution and the treatment was repeated twice at $0 \mathrm{~h}$ and $24 \mathrm{~h}$ later. The induction triggered the reporter gene expression at the whole plant level, GUS activity was detectable in leaves $24 \mathrm{~h}$ after application of TA and 72h with both Dex and TA (Fig. 5B). The induced levels again exceeded the GUS activity detected in the pZmUbi::GUS line, however, but only with 
one of the lines tested (65B). The variability in the measurements could be due to segregating plant populations and a small sample size.

Both painting and watering experiments were repeated with a small modification of the treatment done at 2-day intervals and leaves were imaged using a confocal laser scanning microscope to observe induction of the second reporter gene, YFP (Fig. 5C-K). A bright YFP signal was detected 24h after painting the leaves (Fig. 5C) but not watering the plants (Fig. 5D) with Dex. The signal became stronger at 96 hpi with both methods of treatment (Fig. 5F and 5G) and more cells seemed to be expressing YFP with the TA treatment than Dex (Fig. $5 \mathrm{I}$ and $5 \mathrm{~J}$ ). No signal was detected without any induction (Fig. 5E and $5 \mathrm{H}$ ) as in the WT (Fig. 5K).

To summarise, higher levels of GUS activity and YFP expression were obtained by painting the leaves rather than watering the plants and using TA at equivalent concentrations of Dex, suggesting that TA is a more potent inducer. Thus, whole plant and single leaf phenotypes can be assessed after induction using both methods.

\section{Long-term induction of the pOp6/LhGR system has no negative effects on rice plants}

Final experiments were to determine whether there are any undesirable effects due to long term induction of the p0p6/LhGR system in rice. Four-week old plants were watered with either $30 \mathrm{mM}$ Dex or $30 \mathrm{mM}$ TA or a control solution (DMSO) at 2-day intervals for a week (Fig. 6, left) or two weeks and let to recover for further 6 weeks (Fig. 6 , right). The images suggest that the plants grew and developed normally compared to WT plants treated with the control solution.

\section{Discussion}

\section{A construct design: possible reasons for toxicity, leakiness and silencing}

In the publication of Vlad and colleagues (2019) the activator LhGR was codon optimized for use in rice (rcoLhGR) and further "domesticated" to remove all recognition sites for the Golden Gate cloning. However, severe growth defects on $10 \mu \mathrm{M}$ Dex media were observed with all their constructs tested and in all transgenic lines. As addition of isopropyl $\beta$-D-1-thiogalactopyranoside (IPTG) to the growth media reduced the severity of the growth arrest phenotype, the authors concluded that it was a direct consequence of LhGR activity. However, in our work, we used the activator LhGR2 version that was only partially codon optimized for Arabidopsis to eliminate premature polyadenylation events in the Gal4 domain (Rutherford et al., 2005) and we did not observe any undesirable growth defects. Therefore, it is possible that the rice codon optimization rendered the system too efficient and prone to non-specific offtarget binding due to high rcoLhGR protein levels in the growth inhibited lines. In this regard, to optimize efficiency of an expression system it is recommended to maximize the translational efficiency of a gene of interest (Moore et al., 2006). This can be achieved by taking some simple steps such as ensuring that 
the initiation codon conforms to the consensus for efficient initiation in plants (Koziel et al., 1996) and including translation enhancers in the 5 UTR such as the TMV $\Omega$ in our study.

Similarly, the GVG synthetic transcription factor that incorporates the glucocorticoid receptor (GR) ligand binding domain was found to be detrimental when activated in rice (Owerkverk et al., 2001) and other species including Arabidopsis (Kang et al., 1999; tobacco, Amirsadeghi et al., 2009; Lotus japonicus, Andersen et al., 2003). The growth perturbations were caused probably by the GVG activator that was binding to cis-regulatory elements in the plant genome with sequence homology to GAL4 upon Dex activation (Kang et al., 1999). Nevertheless, the system was used successfully in a number of studies in rice (Chern et al., 2013; Nakayama et al., 2013; Onodera et al., 2018, Park et al., 2012; Qu et al., 2009) in which the negative effects were reduced by shortening the time of Dex exposure or selecting lines with low GVG expression levels and mild phenotype that could be used as activator lines.

Moreover, when designing a construct for an inducible system, care must be taken to ensure that enhancers in the promoter that drives the activator do not activate the target promoter (e.g. p0p6; Moore et al., 2006) as such arrangement might result in transgene activation in the absence of inducer. In the p0p6/rcoLhGR constructs (Vlad et al., 2019), the arrangement was not ideal, as the p0p6 promoter was positioned directly next to the $p Z m U b i$ promoter driving the rcoLhGR. Furthermore, two other potent promoters were used on the same T-DNA; the constitutive rice actin promoter ( $p O s A C T$ ) to drive dsRed for identification of transgenic seeds, and another copy of pOSACT or the CaMV $35 \mathrm{~S}$ promoter (for hygromycin-resistance selectable marker) that can also affect the expression of a transgene (Yoo et al., 2005). Curiously, the authors also used an intron in the reporter gene (GUS) placed again right next to the pOp6 promoter and revealed that the presence of the intron had an enhancing effect on GUS activity levels both in the absence and presence of inducer.

Also, it must be recognized that any transgene may be susceptible to post-transcriptional gene silencing (PTGS) if transcripts accumulate to sufficient levels whether it is expressed constitutively or when induced (Moore et al., 2006). Individual reporter genes have a gene-specific threshold of mRNA abundance that will trigger PTGS (Schubert et al., 2004) and the probability of silencing of an inducible transgene locus in increased if the locus is induced (Abranches et al., 2005). In an attempt to minimize gene silencing, in our construct design we tried not to repeat the same sequence more than once, for example we used different polyadenylation signals (namely the octopine synthase terminator Tocs, nopaline synthase terminator Tnos, and T35S; Fig. 1A). However, we were not able to avoid silencing as only $25 \%$ of hygromycin-resistant transgenic lines (T1) displayed GUS activity after Dex induction compared to $50 \%$ of tested transgenic calli (TO). Others described a similar decrease, for example a reporter gene (GUSPlus) was expressed in $84 \%$ of rice calli but only in $25-68 \%$ of adult plants (Wu et al., 2003); and with the XVE system only $50 \%$ of calli derived from inducible T1 seeds showed detectable GFP signal (Okuzaki et al., 2011).

\section{p0p6/LhGR compared with other systems: is there an ideal inducible system for rice?}


The development of chemical-inducible systems for tight control of plant gene expression is a challenging task. There are a number of properties that are required for an ideal system, such as low basal expression level, high inducibility, specificity and dynamic range of response with respect to the inducer. Also, fast response and induction by various methods is desirable. An ideal system should work in several plant species and should not cause any adverse physiological effects in plants by itself or its inducer. The inducer is further required to show high specificity for the transgene, high efficiency at low concentrations and must not be found in target plants.

The p0p6/LhGR system described here is fast, first GUS activity was detected in painted leaves (Fig. 3A) and visible GUS staining in growing root tips (Fig. 3C) after $12 \mathrm{~h}$ of induction and increased up to $72 \mathrm{~h}$ tested. Similarly, with the rcoLhGR activator, $6 \mathrm{~h}$ of induction was sufficient to induce high levels of GUS activity in transgenic calli and reached maximum levels at 24-96h depending on the construct (maximum levels of activity were reached after $24 \mathrm{~h}$ in lines containing constructs with introns in the reporter gene but required 4 days of induction in the intron-less versions) (Vlad et al., 2019). On the other hand, the GVG system tested in rice (Ouwerkerk et al., 2001) required 4 days for the activity to be detected and 2 weeks of induction for GUS activities to reach levels comparable to those conferred by the strong CaMV $35 \mathrm{~S}$ promoter. With the XVE system, a GFP signal was detected in rice calli $48 \mathrm{~h}$ after induction (with $1 \mu \mathrm{M} 17$ $\beta$-estradiol) and reached maximum (with treatment at $25 \mu \mathrm{M}$ ) in 8 days. However, in calli and leaves of transgenic lines the GFP signals were weaker than those in the leaves of p35S::GFP line, only in roots the signals were similar with 17- $\beta$-estradiol treatment at > $10 \mu \mathrm{M}$ (Okuzaki et al., 2011).

The p0p6/LhGR system is very sensitive, $0.01 \mu \mathrm{M}$ Dex was sufficient to induce GUS activity in painted leaves (Fig. 3B) and in roots of in vitro grown seedlings of the p0p6/rcoLhGR (Vlad et al., 2019). Interestingly, in some lines maximum levels of induction were reached with $0.1 \mu \mathrm{M}$ Dex, while others required $10 \mu \mathrm{M}$ Dex to reach levels similar to the constitutive $p Z m U b i$ promoter (Fig. 3B). This makes the p0p6/LhGR system ten-times more sensitive than the GVG system that required minimum of $0.1 \mu \mathrm{M}$ Dex for induction and 1-10 $\mu \mathrm{M}$ for maximum.

The p0p6/LhGR system is tightly regulated and strong, the average fold induction was app. 500 in shoots of plants induced in vitro (Fig. 2C) or in soil by Dex, however, reached 1000-fold induction when plants were treated with TA (Fig. 5). In roots the magnitude of Dex-induction reached at least 1000- to 6000 -fold (Fig. 2B), similar to the values reported for the GVG system (Ouwerkerk et al., 2001). Both the p0p6/LhGR as well as the pOp6/rcoLhGR systems used the same line that constitutively expressed a synthetic GUS variant from Staphylococcus (GUSPlus) under the control of the $p Z m U b i$ promoter as a positive control. The GUSPlus was reported to be ten-fold more active than the enzyme encoded by $E$. coli uidA that was used in the inducible constructs (Broothaerts et al., 2005; Jefferson et al., 2003), suggesting that activation of the p0p6 promoter by the activators is very effective.

The p0p6/LhGR system is inducible by various methods in vitro and in soil-grown plants. Painting the leaves with an inducer solution supplemented with $0.1 \%$ Tween-20 (to help the inducer to penetrate through the protective wax layer) proved to be more effective than subterranean irrigation (watering). It is 
worth to note that using these methods of application, TA proved to be almost twice as more an efficient inducer than Dex (Fig. 5). This could be due to the nature of the inducer as Dex could be more rapidly metabolized or compartmentalized than TA (Aoyama and Chua, 1997). Ouwerkerk and colleagues (2001) reported that Dex was taken up efficiently by roots of mature plants in hydroponics and induced GUS activity throughout the whole plant body.

The major limitation and possible reasons for low levels of target gene induction in rice leaves using the XVE system is the inefficient uptake of estradiol from hydroponics. Interestingly, cut leaf segments placed in liquid MS media with $10 \mu \mathrm{M}$ estradiol showed detectable GFP fluorescence in whole leaf segments within $48 \mathrm{~h}$ (Okuzaki et al., 2011) and similar activation was confirmed by PCR in another study (Hirose et al., 2012). Also, spraying of leaves with the inducer was another effective method of induction (Hirose et al., 2012). The limiting factor therefore seems to be the estradiol uptake that is depending on the roots. The inducer might have been trapped by XVE expressed in roots or diffused and attenuated in leaves. Interestingly, activation by soaking transgenic seeds (Chen et al., 2017) with estradiol solution induced highly efficient site-specific recombination in germinating embryos, resulting in high-level expression of target gene or RNAi cassette in intact rice plants.

The p0p6/LhGR system is not toxic in rice and does not cause any undesirable negative developmental growth effects even at higher concentration of Dex (up to $30 \mu \mathrm{M}$ tested) and after prolonged exposure of the plants (Fig. 6). However, for the long-term induction through soil, DMSO should be avoided and ethanol should be used as a solvent for the inducers to prevent accumulation of DMSO in the soil over several weeks of watering (Moore et al., 2006; Samalova et al., 2019). Very unexpected were the findings of Vlad and colleagues (2019) that the p0p6/rcoLhGR transgenic plants manifested severe developmental perturbations when grown on concentrations $>0.1 \mu \mathrm{M}$ Dex. The direct cause of these growth defects is not known, but the authors suggested that the rice genome contains sequences with high similarity to the lacOp sequence, suggesting non-specific activation of endogenous genes by Dex induction. Although, the pOp promoter was not activated by endogenous factors in maize (Moore et al., 2006; Segal et al., 2003) it is possible that the rice-codon optimization rendered the system too efficient. The off-target effects can be minimized by quenching with IPTG that acts as an effective antagonist for the Lacl DNA binding domain in LhGR (Craft et al., 2005).

The GVG system has been shown to generate growth defects in rice (Owerkverk et al., 2001) and other species (Kang et al., 1999; Andersen et al., 2003; Amirsadeghi et al., 2009) but the reason for this is not clear as each domain of the molecule has been used successfully in other expression systems. However, one possibility is that GVG requires higher expression levels to achieve full promoter activation, resulting perhaps in squelching or non-specific binding to $C C G(N 11) C G G$ sequences in the genome (Moore et al., 2006). Apparently, the GVG level was not limiting for target-gene activation (Aoyama and Chua, 1997), therefore, selection of plants with a mild GVG phenotype in combination with optimized induction conditions was recommended for the system applications. Although, the XVE system had no effect on growth, all tested lines including controls grown in hydroponics presented a different root pattern with 
oestradiol treatment so the system does not appear to be suitable for root morphology studies (Okuzaki et al., 2011).

\section{Methods}

\section{Construct preparation}

To prepare the pVecLhGR2 construct (Fig. 1A, Fig. S1), first, the LhGR2 and the octopine synthase terminator (Tocs) sequences were amplified by polymerase chain reaction (PCR) using vector pOpln2 (Samalova et al., 2019) as the template. The two PCR fragments, LhGR2 (forward primer P1: 5'AAAAAGGTACCATGGCTAGTGAAGCTCGAAAAACAAAG-3'; reverse primer P2: 5'GCATATCTCATTAAAGCAGGACTCTAGTTCACTCCTTCTTAGGGTTAGGTGGAGTATC-3') and Tocs (forward primer P3: 5'-TACTCCACCTAACCCTAAGAAGGAGTGAACTAGAGTCCTGCTTTAATGAGATATGC-3'; reverse primer P4: 5'- AAAAAGGTACCCTAAGGCGCGCCGTTGTCG CAAAATTCGCCCTGGACCC - 3') were joined together by overlapping PCR with primers $\mathrm{P} 1$ and $\mathrm{P} 4$ and cloned into the unique $K p n$ site of the binary rice overexpression vector pVec8-KpnIOX (Kim and Dolan, 2016). Secondly, an Ascl fragment containing the bidirectional pOp6 operator array together with $T M V \Omega$ translation enhancer sequences driving expression of YFP and GUS (including 35S terminator sequence) reporters of the pOpln2YFP vector (generated by M. Kalde, I. Moore lab) was cloned into a unique Ascl site created in the previous cloning step. The correct orientation of the fragment as well as the LhGR2 in the final pVecLhGR2 vector was confirmed by sequencing.

\section{Plant material and growth conditions}

Seeds of Oryza sativa spp. japonica cultivar Kitaake were obtained from the International Rice Research Institute, Los Banos, Philippines and kindly provided by Peng Wang (Chinese Academy of Sciences, Shanghai, China). To generate stable transgenic rice lines, we used Agrobacterium-mediated transformation of Oryza sativa spp. japonica cultivar Kitaake. Calli were induced from dehulled mature seeds before co-cultivation with $A$. tumefaciens strain EHA105 transformed with the pVecLhGR2 construct. Callus transformation and seedling regeneration were performed at $28{ }^{\circ} \mathrm{C}$ according to a protocol modified from Toki et al., (2006) as described by Vlad et al., 2019. Independent pVecLhGR2 lines were tested together with positive pZmUbi:: GUS (Vlad et al., 2019) and negative wild-type (WT) controls. Soil-grown plants were cultivated in a greenhouse at $28-30{ }^{\circ} \mathrm{C}$ with a $16 \mathrm{~h} / 8 \mathrm{~h}$ photoperiod.

\section{Methods of steroid induction - in vitro, painting and watering}

Dexamethasone (Dex) was prepared as a $30 \mathrm{mM}$ stock solution in dimethyl sulfoxide (DMSO) and stored at $-20^{\circ} \mathrm{C}$. Similarly, we prepared stock solutions of betamethasone (Bet), deoxycorticosterone (Doc), fludrocortisone acetate (Flu), prednisone (Pre), prednisolone (Plo) and triamcinolone acetonide (TA), all 
purchased from Sigma-Aldrich. For each treatment, either a glucocorticoid (induction) or the equivalent volume of DMSO (control) were added to obtain desired concentration.

For application in vitro, seedlings were grown on half strength Murashige and Skoog (1962) medium (1/2 MS medium) supplemented with $15 \mathrm{~g} / \mathrm{l}$ sucrose and with or without agar. Dex was added to the medium after autoclaving to obtain final working concentration, typically $30 \mu \mathrm{M}$ Dex. Shoots and leaf cuttings were also induced in distilled water supplemented with $0.1 \%$ Tween-20 and a glucocorticoid inducer (10$30 \mu \mathrm{M})$.

For induction of soil grown plants, 4-week-old rice plants were either watered with $50 \mathrm{ml}$ of $30 \mu \mathrm{M}$ Dex or $30 \mu \mathrm{M}$ TA or newly developed leaves (3rd leaf) were painted on both sides with $10 \mu \mathrm{M}$ Dex or $10 \mu \mathrm{M}$ TA solution supplemented with $0.1 \%$ Tween-20. The treatments were repeated at 2-day intervals for one or two weeks.

\section{Analysis of ß- glucuronidase (GUS) reporter activity}

Histochemical GUS staining and fluorometric GUS assay was performed according to Jefferson (1987) at $37^{\circ} \mathrm{C}$. Extracts were prepared from ground snap-frozen samples; the protein content was determined spectrophotometrically using Bio-Rad Protein Assay (Bio-Rad laboratories). The fluorogenic reaction was carried out in 96 well-plates using protein extraction buffer supplemented with 4-methylumbelliferyl ß-Dglucuronide (4-MUG) as a substrate. A standard curve of 4-MU was used to calculate the amount of 4$\mathrm{MU} /$ unit of time. Activity was calculated from three technical replicates and expressed in pmoles 4$\mathrm{MU} / \mathrm{min} / \mu \mathrm{g}$ protein.

\section{Detection of YFP fluorescence}

The YFP signal was detected using a Zeiss LSM 510 Meta confocal laser-scanning microscope with 514nm excitation from an Argon laser and a BP535-590IR emission filter.

\section{Abbreviations}

Dex: Dexamethasone; LhGR: chimeric transcription factor; p0p6: inducible promoter based on the lac operators; lacOp: lac operators; CaMV: cauliflower mosaic virus $35 \mathrm{~S}$ promoter; $p Z m U b i$ : maize ubiquitin promoter containing an intron; $T M V$ : tobacco mosaic virus; $\Omega$ : omega translation enhancers; uidA: gene encoding b-glucuronidase or GUS; YFP: yellow fluorescent protein; HYG: hygromycin phosphotransferase; $1 \frac{1}{2}$ MS: half strength Murashige and Skoog medium; DMSO: dimethyl sulfoxide; TA: triamcinolone acetonide; 4-MU: 4-methyl umbelliferone; 4-MUG: 4-methylumbelliferyl ß-Dglucuronide; rco: rice codon optimized; IPTG: isopropyl $\beta$-D-1-thiogalactopyranoside

\section{Declarations}

\section{ETHICS APPROVAL AND CONSENT TO PARTICIPATE}


Not applicable.

\section{CONSENT FOR PUBLICATION}

Not applicable.

\section{AVAILABILITY OF DATA AND MATERIAL}

All data generated or analysed during this study are included in this published article.

\section{COMPETING INTEREST}

No conflicts of interest declared.

\section{FUNDING}

Not applicable.

\section{AUTHORS`CONTRIBUTIONS}

MS: Conceptualization; Investigation; Writing IM: Conceptualization; Supervision; Funding

\section{ACKNOWLEDGEMENTS}

I would like to dedicate this publication to my colleague and friend lan Moore, and his beloved family, without whom science is much less fun. I want to thank to Peng Wang (Chinese Academy of Sciences, Shanghai, China) for the control pZmUbi::GUS line and the rice transformation protocol; to Chul Min Kim (University of Oxford) for the pVec8-KpnIOX vector and to Monika Kalde (University of Oxford) for the pOpIn2YFP vector. I am grateful to Molly Dewey for her suggested word changes.

Significance statement: The non-monocot codon-optimized version of the dexamethasone inducible pOp6/LhGR system does not cause severe developmental perturbations in rice plants.

\section{References}

1. Abranches R, Shultz RW, Thompson WF, Allen, GC. Matrix attachment regions and regulated transcription increase and stabilize transgene expression. Plant Biotechnol J. 2005;3:535-43.

2. Amirsadeghi S, McDonald AE, Vanlerberghe CC. A glucocorticoid-inducible gene expression system can cause growth defects in tobacco. Planta. 2009;226:453-63.

3. Andersen SU, Cvitanich C, Hougaard BK, Roussis A, Gronlund M, Jensen DB, Frokjaer LA, Jensen EO. The glucocorticoid-inducible GVG system causes severe growth defects in both root and shoot of the model legume Lotus japonicus. Mol Plant Microbe Interact. 2003;16:1069-76.

4. Aoyama T, Chua N-H. A glucocorticoid-mediated transcriptional induction system in transgenic plants. Plant J. 1977;11:605-12. 
5. Broothaerts W, Mitchell HJ, Weir B, Kaines S, Smith LMA, Yang W, Mayer JE, Roa-Rodríguez C, Jefferson RA. Gene transfer to plants by diverse species of bacteria. Nature. 2005;433:629-33.

6. Caddick MX, Greenland AJ, Jepson I, Krause K-P, Qu N, Riddell KV, Salter MG, Schuch W, Sonnewald $\mathrm{U}$, Tomsett AB. An ethanol inducible gene switch for plants used to manipulate carbon metabolism. Nat Biotechnol. 1998;16:177-80.

7. Chen ZJ, Cheng QQ, Hu CQ, Guo XR, Chen ZQ, Lin Y, Hu TJ, Bellizzi M, Lu GD, Wang GL, Wang ZH, Chen SB, Wang ZH. A Chemical-induced, seed-soaking sctivation procedure for regulated gene expression in rice. Front Plant Sci.2017;8:1447.

8. Chern M, Bai W, Chen X, Canlas PE, Ronald PC. Reduced expression of glycolate oxidase leads to enhanced disease resistance in rice. Peer J. 2013;1:e28.

9. Craft J, Samalova M, Baroux C, Townley H, Martinez A, Jepson I, Tsiantis M, Moore I. New pOp/LhG4 vectors for stringent glucocorticoid-dependent transgene expression in Arabidopsis. Plant $\mathrm{J}$. 2005;41:899-918.

10. Corrado G, Karali M. Inducible gene expression systems and plant biotechnology. Biotechnol Adv. 2009;27:733-43.

11. Du DX, Jin RC, Guo JJ, Zhang FD. Construction of marker-free genetically modified maize using a heat-inducible auto-excision vector. Genes. 2019;10:374.

12. Eva C, Teglas F, Zelenyanszki H, Tamas C, Juhasz A, Meszaros K, Tamas L. Cold inducible promoter driven Cre-lox system proved to be highly efficient for marker gene excision in transgenic barley. $J$ Biotechnol. 2018;265:15-24.

13. Goh H-H, Sloan J, Dorca-Fornell C, Fleming A. Inducible repression of multiple expansin genes leads to growth suppression during leaf development. Plant Physiol. 2012;159:1759-70.

14. Helliwell EE, Wang Q, Yang Y. Transgenic rice with inducible ethylene production exhibits broadspectrum disease resistance to the fungal pathogens Magnaporthe oryzae and Rhizoctonia solani. Plant Biotechnol J. 2013;11:33-42.

15. Hirose T, Mizutani R, Mitsui T, Terao T. A chemically inducible gene expression system and its application to inducible gene suppression in rice. Plant Prod Sci. 2012;15:73-78.

16. Honma Y, Yamakawa T. High expression of GUS activities in sweet potato storage roots by sucroseinducible minimal promoter. Plant Cell Rep. 2019;38:SI 1417-26.

17. Jefferson RA. Assaying chimeric genes in plants: the GUS gene fusion system. Plant Mol Biol Rep. 1987;5:387-405.

18. Jefferson RA, Kilian A, Wilson KJ, Keese PK. Microbial $\beta$-glucuronidase genes, gene products and uses thereof. US Patent. 2003;391:547.

19. Ji X, Si XM, Zhang Y, Zhang HW, Zhang F, Gao CX. Conferring DNA virus resistance with high specificity in plants using virus-inducible genome-editing system. Genome Biol. 2018;19:197.

20. Jiang WZ, Zhou, HB, Bi, HH, Fromm M, Yang B, Weeks DP. Demonstration of CRISPR/Cas9/sgRNAmediated targeted gene modification in Arabidopsis, tobacco, sorghum and rice. Nucl Acids Res. 
2013;41:e188.

21. Kang HG, Fang Y, Singh KB. A glucocorticoid-inducible transcription system causes severe growth defects in Arabidopsis and induces defense-related genes. Plant J. 1999;20:127-33.

22. Khattri A, Nandy S, Srivastava V. Heat-inducible Cre-lox system for marker excision in transgenic rice. J Biosci. 2011;36:37-42.

23. Kim CM, Dolan L. ROOT HAIR DEFECTIVE SIX-LIKE class I genes promote root hair development in the grass Brachypodium distachyon. PLoS Genet. 2016;12:e1006211.

24. Koo JC, Asurmendi S, Bick J, Woodford-Thomas T, Beachy RN. Ecdysone agonist-inducible expression of a coat protein gene from tobacco mosaic virus confers viral resistance in transgenic Arabidopsis. Plant J. 2004;37:439-48.

25. Kopertekh L, Krebs E, Guzmann F. Improvement of conditional Cre-lox system through application of the regulatory sequences from Cowpea mosaic virus. Plant Biotechnol. Rep. 2018;12:127-137.

26. Koziel MG, Carozzi NB, Desai N. Optimizing expression of transgenes with an emphasis on posttranscriptional events. Plant Mol Biol. 1996;32:392-405.

27. Liu SM, Yoder JI. Chemical induction of hairpin RNAi molecules to silence vital genes in plant roots. Sci Rep. 2016;6:37711.

28. Meszaros K, Eva C, Kiss T, Banyai J, Kiss E, Teglas F, Lang L, Karsai I, Tamas L. Generating markerfree transgenic wheat using minimal gene cassette and cold-inducible Cre/lox system. Plant Mol Biol Rep. 2015;33:1221-31.

29. Mitsuhara I, Ugaki M, Hirochika H, Ohshima M, Murakami T, Gotoh Y, Katayose Y, Nakamura S, Honkura R, Nishimiya S, Ueno K, Mochizuki A, Tanimoto H, Tsugawa H, Otsuki Y, Ohashi Y. Efficient promoter cassettes for enhanced expression of foreign genes in dicotyledonous and monocotyledonous plants. Plant Cell Physiol. 1996;37:49-59.

30. Moore I, Samalova M, Kurup S. Transactivated and chemically inducible gene expression in plants. Plant J. 2006;45:651-83.

31. Murashige T, Skoog F. A revised medium for rapid growth and bioassays with tobacco tissue. Physiol Plant. 1962;15:493-97.

32. Nakashima K, Tran L.-SP, Van Nguyen D, Fujita M, Maruyama K, Todaka D, Ito Y, Hayashi N, Shinozaki $\mathrm{K}$, Yamaguchi-Shinozaki K. Functional analysis of a NAC-type transcription factor OsNAC6 involved in abiotic and biotic stress-responsive gene expression in rice. Plant J. 2007;51:617-30.

33. Nakayama A, Fukushima S, Goto S, Matsushita A, Shimono M, Sugano S, Jiang C-J, Akagi A, Yamazaki M, Inoue $\mathrm{H}$, Takatsuji, $\mathrm{H}$. Genome-wide identification of WRKY45-regulated genes that mediate benzothiadiazole-induced defense responses in rice. BMC Plant Biol. 2013;13:150.

34. Nandy S, Pathak B, Zhao S, Srivastava V. Heat-shock-inducible CRISPR/Cas9 system generates heritable mutations in rice. Plant Direct. 2019;3:UNSP145.

35. Okuzaki A, Konagaya K-I, Nanasato Y, Tsuda M, Tabei Y. Estrogen-inducible GFP expression patterns in rice (Oryza sativa L.). Plant Cell Rep. 2011;30:529-38. 
36. Onodera H, Shingu S, Ohnuma M, Horie T, Kihira M, Kusano H, Teramura H, Shimada H.

Establishment of a conditional TALEN system using the translational enhancer dMac3 and an inducible promoter activated by glucocorticoid treatment to increase the frequency of targeted mutagenesis in plants. PLOS One. 2018;13:e0208959.

37. Ouwerkerk PBF, de Kam RJ, Hodge JHC, Meijer AH. Glucocorticoid-inducible gene expression in rice. Planta. 2001;213:370-78.

38. Padidam M, Gore M, Lu DL, Smirnova O. Chemical-inducible, ecdysone receptor-based gene expression system for plants. Trans Res. 2003;12:101-109.

39. Park C-J, Canlas PE, Ronald PC. Establishment of glucocorticoid-mediated transcriptional induction of the rice Xa21 pattern recognition receptor. J Plant Biol. 2012;55:43-49.

40. Qu S, Jeon J-S, Ouwerkerk PBF, Bellizzi M, Leach J, Ronald P, Wang G-L. Construction and application of efficient Ac-Ds transposon tagging vectors in rice. J Integ Plant Biol. 2009;51:982-92.

41. Quilis J, Lopez-Garcia B, Meynard D, Guiderdoni E, San Segundo B. Inducible expression of a fusion gene encoding two proteinase inhibitors leads to insect and pathogen resistance in transgenic rice. Plant Biotechnol. J. 2014;12:367-77.

42. Rerksiri W, Zhang X, Xiong H, Chen X. Expression and promoter analysis of six heat stress-inducible genes in rice. Sci World J. 2013;397-401.

43. Rose AB. The effect of intron location on intron-mediated enhancement of gene expression in Arabidopsis. Plant J. 2004;40:744-51.

44. Roslan HA, Salter MG, Wood CD, White MRH, Croft KP, Robson F, Coupland G, Doonan J, Laufs P, Tomsett AB, Caddick MX. Characterization of the ethanol-inducible alc gene-expression system in Arabidopsis thaliana. Plant J. 2001;28:225-35.

45. Rossignol P, Orbovic V, Irish VF. A dexamethasone-inducible gene expression system is active in citrus plants. Sci Hortic. 2014;172:47-53.

46. Rutherford S, Brandizzi F, Townley H, Craft J, Wang Y, Jepson I, Martinez A, Moore I. Improved transcriptional activators and their use in mis-expression traps in Arabidopsis. Plant J. 2005;43:76988.

47. Salter MG, Paine JA, Riddell KV, Jepson I, Greenland AJ, Caddick M, Tomsett AB. Characterisation of the ethanol-inducible alc gene expression system for transgenic plants. Plant J. 1998;16:127-32.

48. Samalova M, Brzobohaty B, Moore I. p0p6/LhGR: a stringently regulated and highly responsive dexamethasone-inducible gene expression system for tobacco. Plant J. 2005;41:919-35.

49. Samalova M, Kirchhelle C, Moore I. Universal methods for transgene induction using the dexamethasone-inducible transcription activation system pOp6/LhGR in Arabidopsis and other plant species. Curr Protoc Plant Biol. 2019;e20089.

50. Samalova M, Elsayad K, Melnikava A, Peaucelle A, Gahurova E, Gumulec J, Spyroglou I, Zemlyanskaya EV, Ubogoeva EV, Hejatko J. Expansin-controlled cell wall stiffness regulates root growth in Arabidopsis. bioRxiv 2020;2020.06.25.170969. 
51. Schubert D, Lechtenberg B, Forsbach A, Gils M, Bahadur S, Schmidt R. Silencing in Arabidopsis TDNA transformants: the predominant role of a gene specific RNA sensing mechanism versus position effects. Plant Cell. 2004;16:2561-72.

52. Schurholz AK, Lopez-Salmeron V, Li ZN, Forner J, Wenzl C, Gaillochet C, Augustin S, Barro AV, Fuchs M, Gebert M, Lohmann JU, Greb T, Wolf, S. A comprehensive toolkit for inducible, cell type-specific gene expression in Arabidopsis. Plant Physiol. 2018;178:40-53.

53. Segal G, Song R, Messing J. A new opaque variant of maize by a single dominant RNA-interferenceinducing transgene. Genetics. 2003;165:387-97.

54. Siligato R, Wang X, Yadav SR, Lehesranta S, Ma GJ, Ursache R, Sevilem I, Zhang J, Gorte M, Prasad K, Wrzaczek M, Heidstra R, Murphy A, Scheres B, Mahonen AP. Multisite Gateway-compatible cell type-specific gene-inducible system for plants. Plant Physiol. 2016;170:627-41.

55. Sreekala C, Wu L, Gu K, Wang D, Tian D, Yin Z. Excision of a selectable marker in transgenic rice (Oryza sativa L.) using a chemically regulated Cre/loxP system. Plant Cell Rep. 2005;24:86-94.

56. Sun F, Qi W, Qian X, Wang Q, Yang M, Dong X, Yang J. Investigating the role of OsPDCD5, a homolog of the mammalian $P D C D 5$, in programmed cell death by inducible expression in rice. Plant Mol Biol Rep. 2012;30:87-98.

57. Timerbaev V, Dolgov S. Functional characterization of a strong promoter of the early light-inducible protein gene from tomato. Planta. 2019;250:1307-23.

58. Toki S, Hara N, Ono K, Onodera H, Tagiri A, Oka S, Tanaka H. Early infection of scutellum tissue with agrobacterium allows high-speed transformation of rice. Plant J. 2006;47:969-76.

59. Vlad D, Abu-Jamous B, Wang P, Langdale J. A modular steroid-inducible gene expression system for use in rice. BMC Plant Biol. 2019;19:426.

60. Wang X, Ye L, Lyu M, Ursache R, Loytynoja A, Mahonen AP. Aa inducible genome editing system for plants. Nat Plants. 2020;6:766-72.

61. Wielopolska A, Townley H, Moore I, Waterhouse P, Helliwell C. A high-throughput inducible RNAi vector for plants. Plant Biotechnol J. 2005;3:583-90.

62. Woo HJ, Qin Y, Park SY, Park SK, Cho YG, Shin KS, Lim MH, Cho HS. Development of selectable marker-free transgenic rice plants with enhanced seed tocopherol content through FLP/FRT-mediated spontaneous auto-excision. PLOS One. 2015;10:e0132667.

63. Wu CY, Li XJ, Yuan WY, Chen GX, Kilian A, Li J, Xu C, Li XH, Zhou DX, Wang S, Zhang Q. Development of enhancer trap lines for functional analysis of the rice genome. Plant J. 2003;35:418-27.

64. Yoo SY, Bomblies K, Yoo SK, Yang JW, Choi MS, Lee JS, Weigel D, Ahn JH. The 35S promoter used in a selectable marker gene of a plant transformation vector affects the expression of the transgene. Planta. 2005;221:523-30.

65. Zhang H, Zhang JS, Wei PL, Zhang BT, Gou F, Feng ZY, Mao YF, Yang L, Zhang H, Xu NF, Zhu JK. The CRISPR/Cas 9 system produces specific and homozygous targeted gene editing in rice in one generation. Plant Biotechnol J. 2014;12:797-807. 
66. Zuo J, Niu Q-W, Chua N-H. An estrogen receptor-based transactivator XVE mediates highly inducible gene expression in transgenic plants. Plant J. 2000;24:265-73.

\section{Tables}

Table 1: Number of putative transformants and generated transgenic lines tested for hygromycin (HYG) resistance and induction of GUS activity in subsequent generations.

\begin{tabular}{|cccc|}
\hline Generation & No. plants tested & HYG resistant & GUS staining \\
\hline T0 & 120 & n.d. & 63 \\
T1 & 166 & 33 & 8 \\
T2 & 5 & 5 & 5 \\
\hline
\end{tabular}

\section{Figures}




\section{A pVecLhGR2}

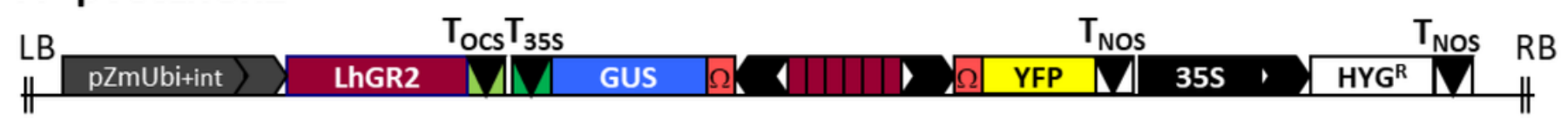

B Lines:
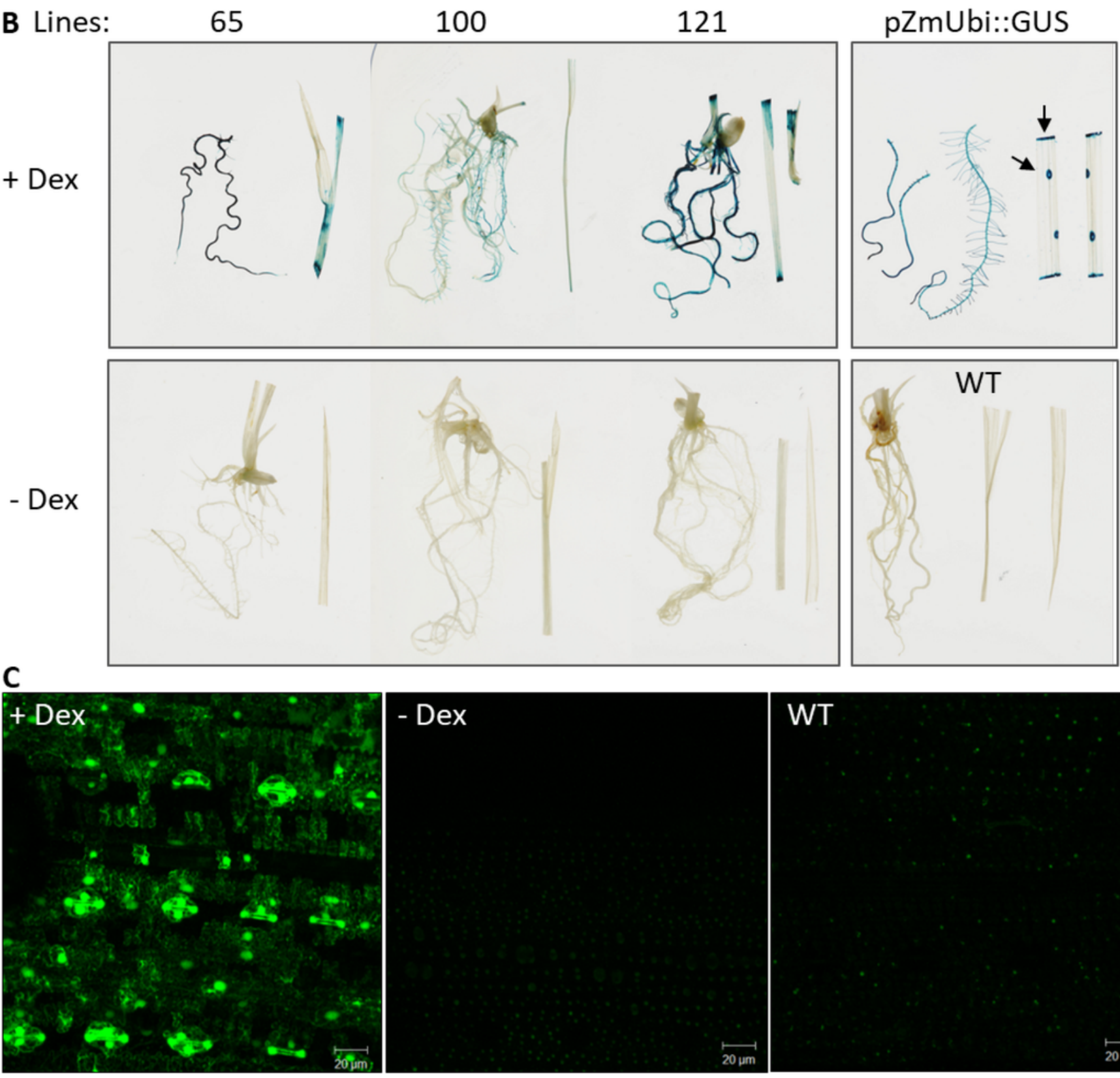

WT

\section{Figure 1}

The p0p6/LhGR system in rice: a proof of principle (A) A schematic representation of the pVecLhGR2 construct that contains the Arabidopsis codon604 optimized GAL4 sequence (Rutherford et al., 2005) of the transcriptional activator LhGR2 driven by a pZmUbi promoter (containing an intron), a bidirectional p0p6 promotor version with two TMV $\Omega$ translation enhancers driving two reporter genes - uidA (encoding $\beta$-glucuronidase; GUS) and a yellow fluorescence protein (YFP), and a pCaMV35S::HYG selectable marker cassette conferring hygromycin resistance. The construct was assembled as detailed in Supplementary 
Figure S1. (B) Twelve-day-old rice seedlings of three independent transgenic lines (number 65, 100 and 121) histochemically stained for GUS activity that was induced by seedling transfer onto $\frac{1}{2} \mathrm{MS}$ plates containing $30 \mu \mathrm{M}$ Dex or control DMSO (-Dex) for 6 days. Seedlings of pZmUbi::GUS and wild-type (WT) were included as controls. The arrows point to an example of damaged cells/ cuts where the GUS staining substrate (5-bromo-4-chloro-3-indolyl glucuronide; X-Gluc) penetrated inside the cells. (C) Leaves of 4-week-old plant (line 121) were painted with $30 \mu \mathrm{M}$ Dex or control solution (-Dex) and imaged using a confocal laser scanning microscope $96 \mathrm{~h}$ later to detect YFP fluorescence. WT was included as a control for autofluorescence. The scale bar is $2 \mu \mathrm{m}$.

A

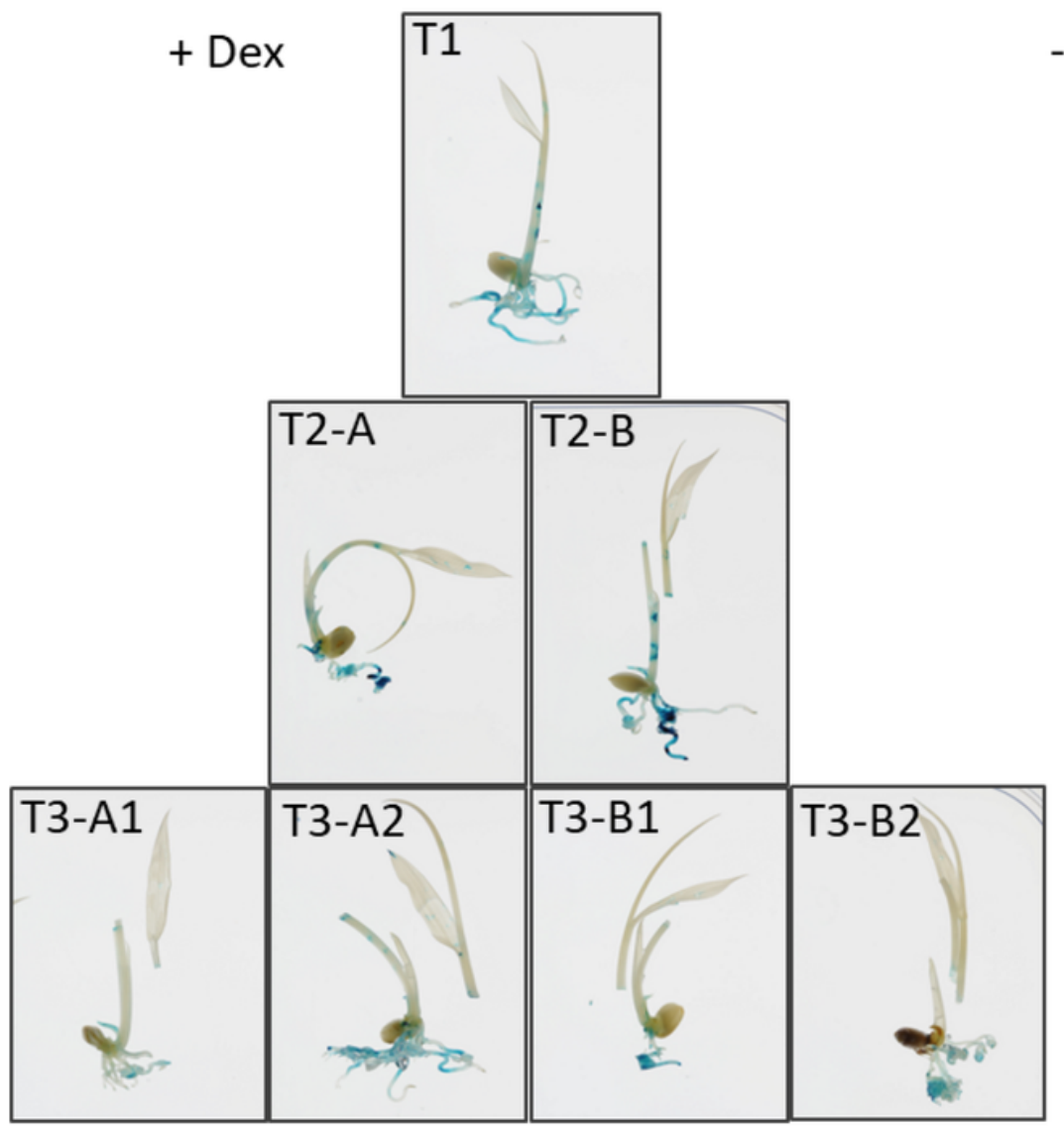

- Dex

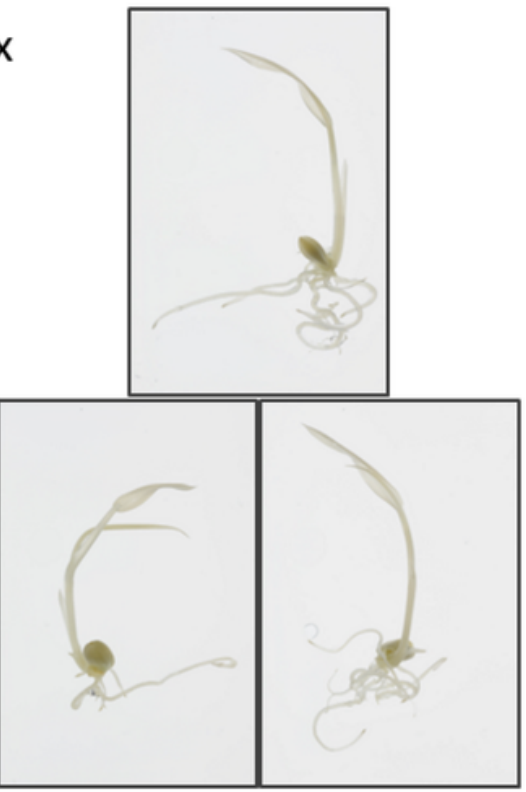

pZmUbi::GUS

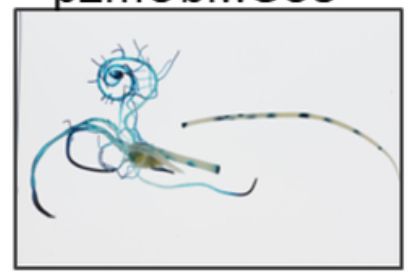

B Roots

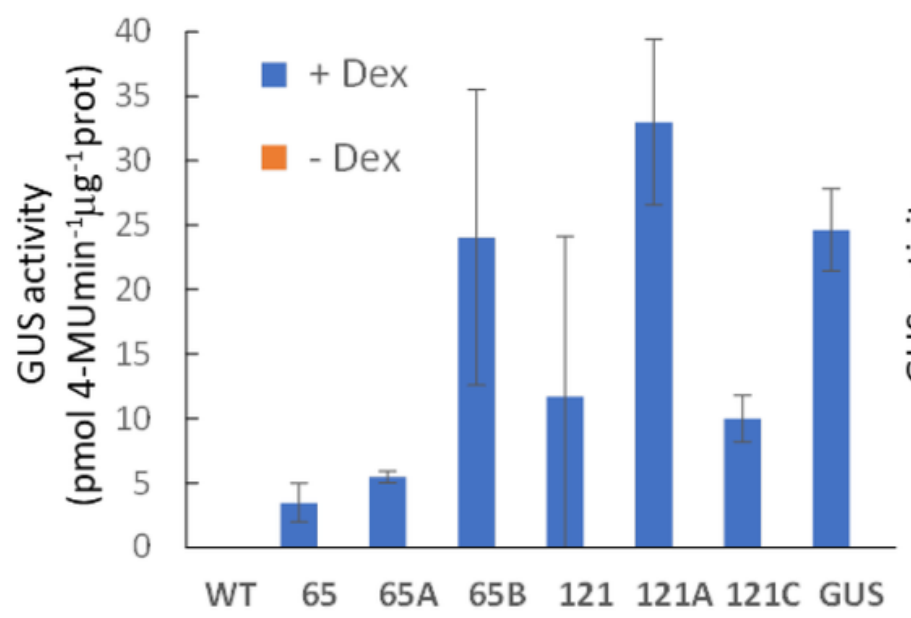

C Shoots

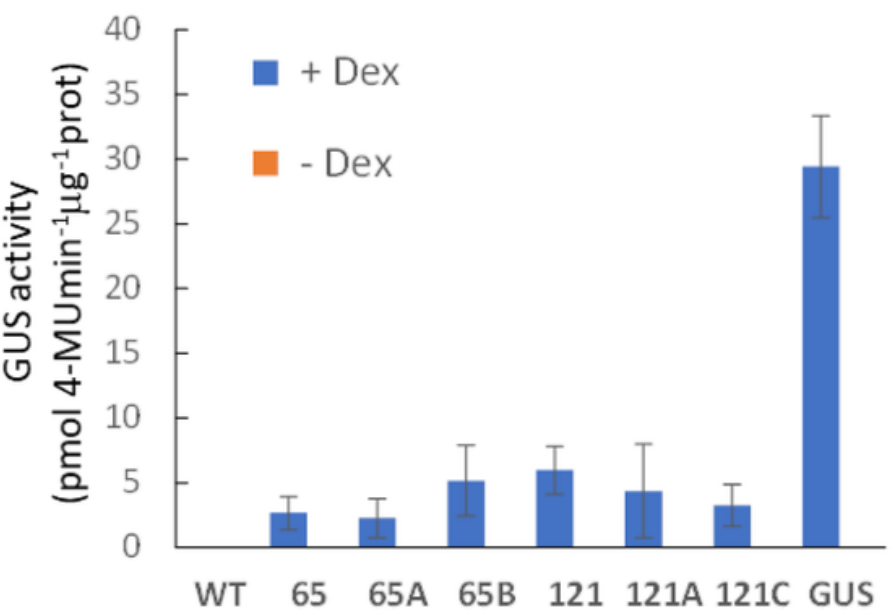




\section{Figure 2}

Reliability of inducible expression in subsequent generations and GUS activity in rice (A) Ten-day-old rice seedlings (line 65) of generation T1, T2 (A and B) and T3 (A1, A2 and B1, B2) histochemically stained for GUS activity induced by germinating and growing the seedlings on $1 / 2 \mathrm{MS}$ plates containing $30 \mu \mathrm{M}$ Dex or control DMSO (-Dex) for 10 days. Seedlings of pZmUbi::GUS were included as a control for the GUS staining. (B, C) GUS activity determined fluorometrically in roots (B) and shoots (C) of 7-day-old rice seedlings (lines 65 and 121, T1 and T2) germinated and grown on $1 / 2$ MS plates containing $30 \mu \mathrm{M}$ Dex or control DMSO (-Dex) for 7 days. Seedlings of WT and pZmUbi::GUS were included as controls. The error bars represent SD. 
A

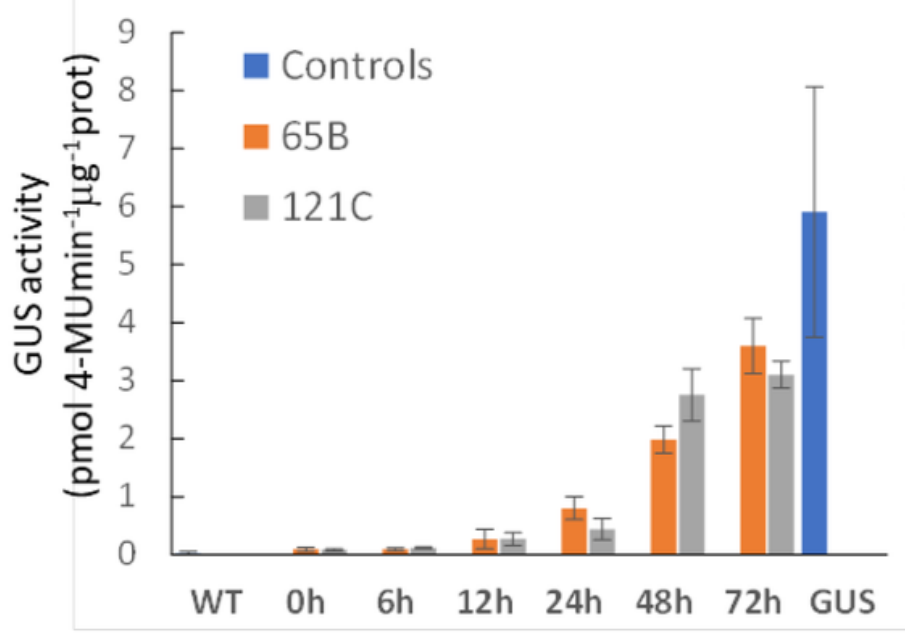

B

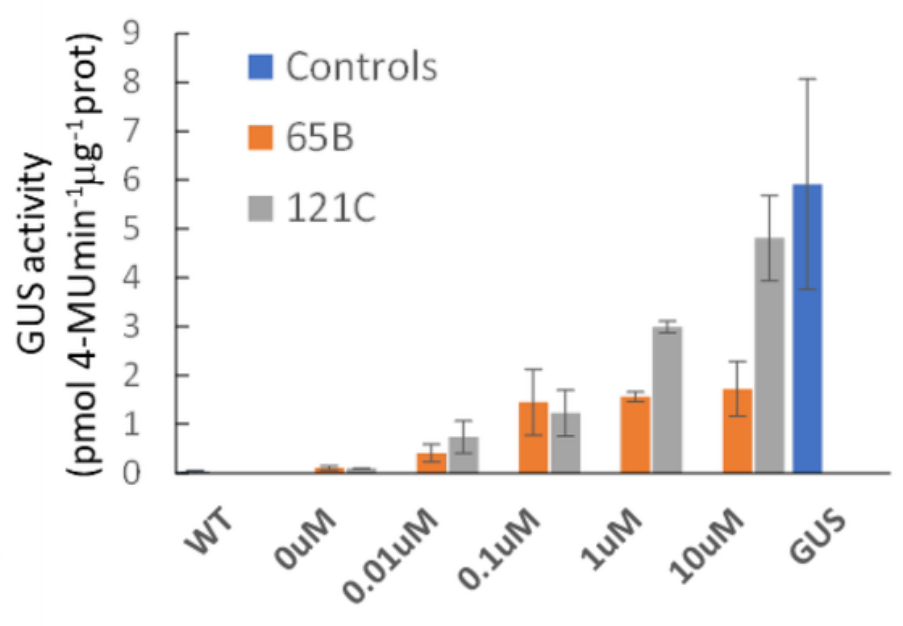

C

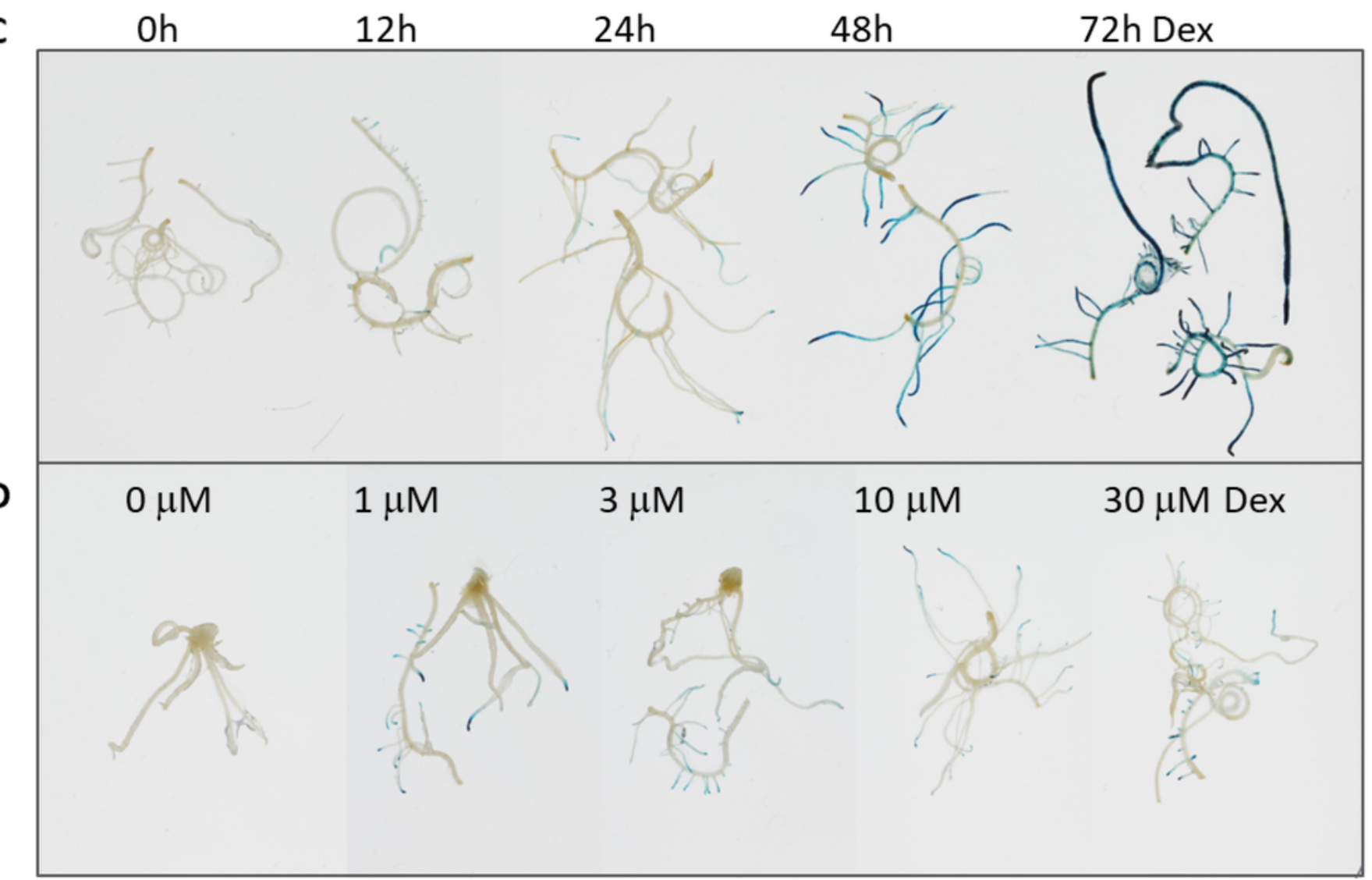

\section{Figure 3}

Time course accumulation of induced GUS activity and dose-response of the p0p6/LhGR system in rice GUS activity determined fluorometrically in 7-day-old rice seedlings (lines 65B and 121C) germinated on $1 / 2$ MS plates (vertically placed in the incubator) and then stood up in $15 \mathrm{ml}$ Falcon tubes with $2 \mathrm{ml}$ water for further 5 days. The newly developed leaves were induced by painting with water supplemented with $0.1 \%$ Tween-20 and (A) $10 \mu \mathrm{M}$ Dex for $0,6,12,24,48$ and $72 \mathrm{~h}$ or (B) with $0,0.01,0.1,1$ and $10 \mu \mathrm{M}$ Dex for 48h. Seedlings of WT and pZmUbi::GUS were included as controls. The error bars represent SD. (C) Roots 
of 10-day-old seedlings (lines 65A, 121C) were induced in liquid $1 / 2 \mathrm{MS}$ with $30 \mu \mathrm{M}$ Dex for $0,12,24.48$ and $72 \mathrm{~h}$, then histochemically stained for GUS activity. Representative images are shown. (D) Roots of 10-day-old seedlings (lines 65A, 121C) were induced in liquid $1 / 2 \mathrm{MS}$ for $24 \mathrm{~h}$ with $0,1,3,10$ and $30 \mu \mathrm{M}$ Dex, then histochemically stained for GUS activity. Representative images are shown.

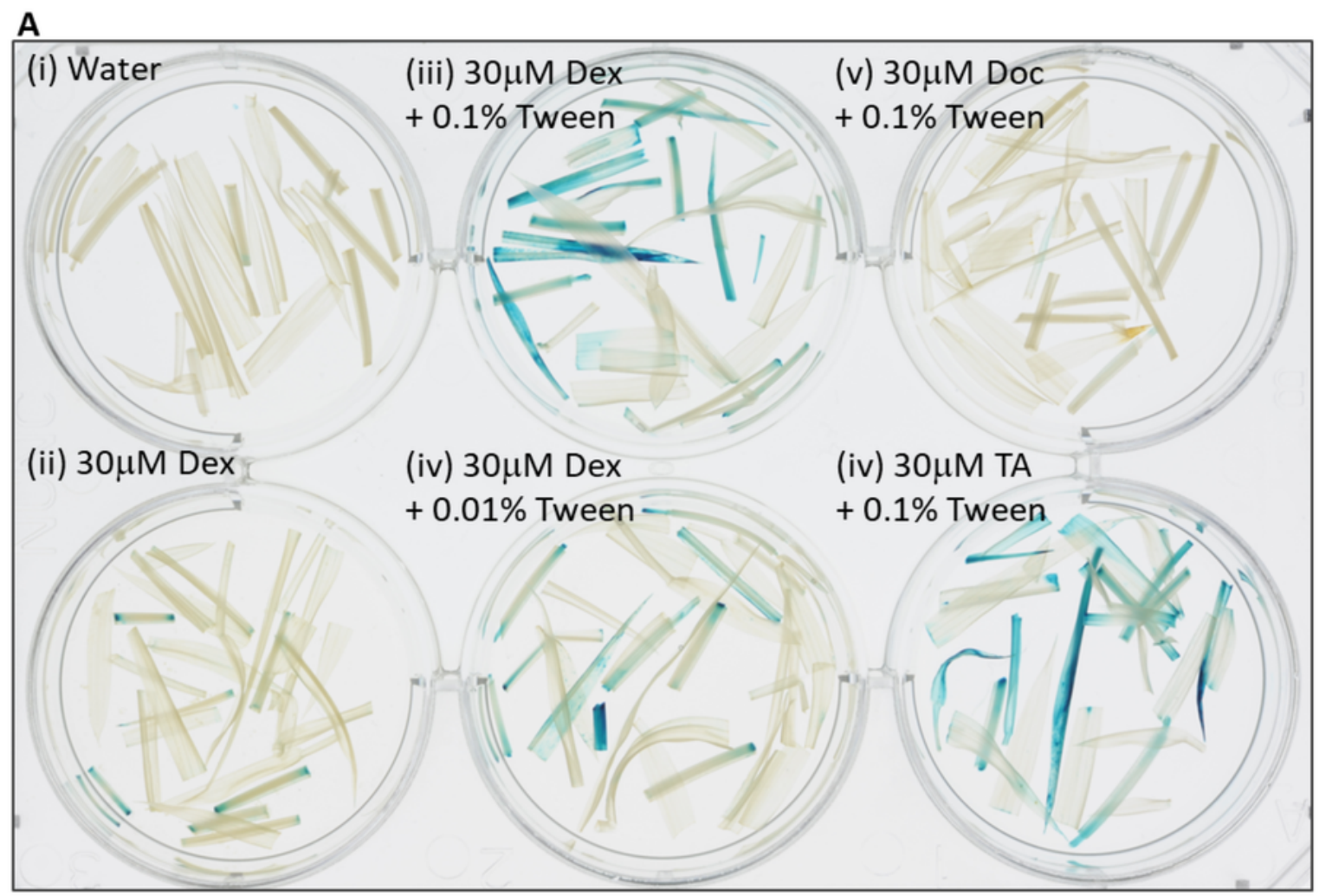

B
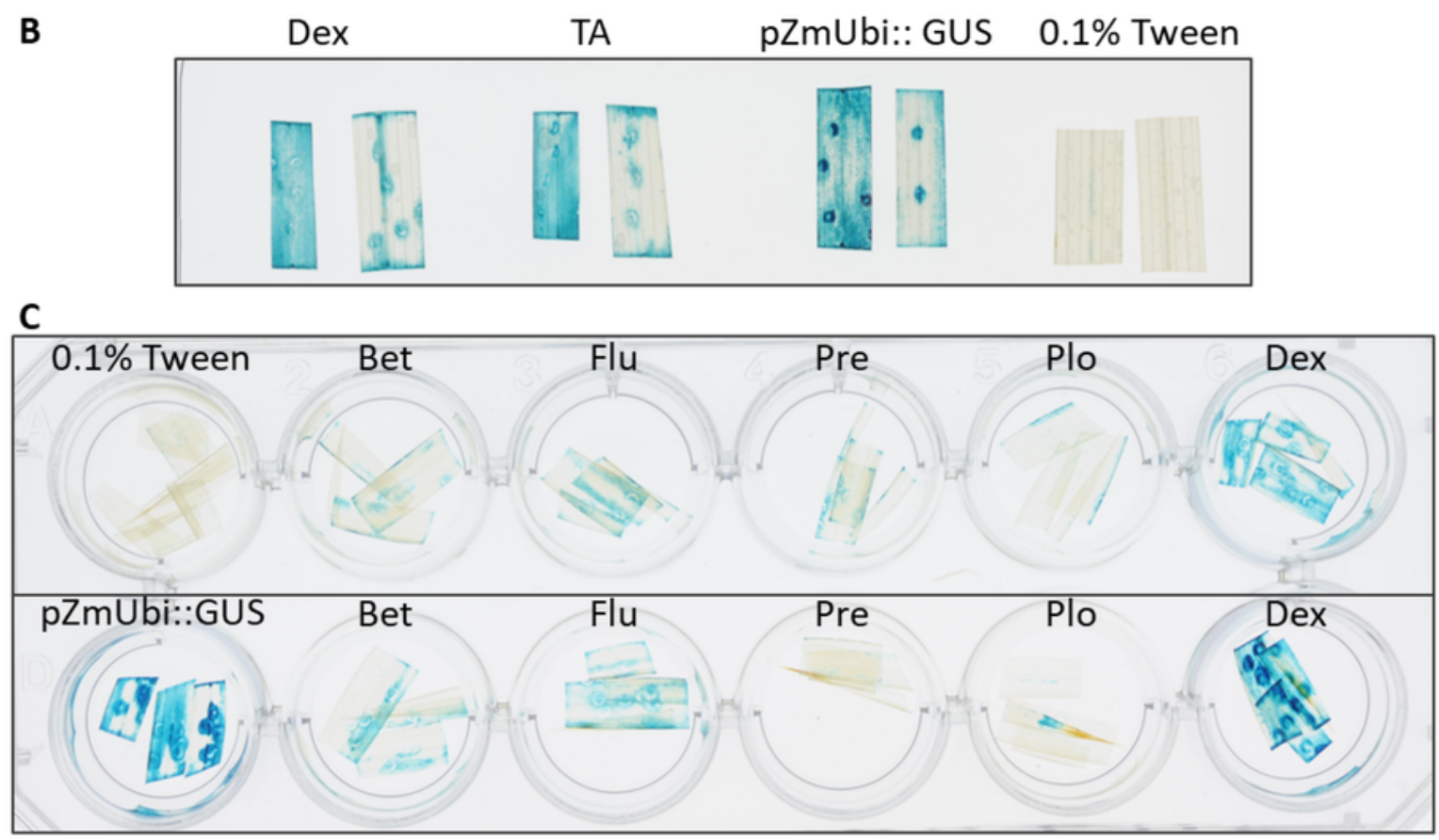

Figure 4 
Optimization of the pOp6/LhGR induction in rice by testing different steroid inducers (A) Shoots of 10 day-old seedlings (lines 65A, 121C) were induced in water supplemented with either $0.01 \%$ or $0.1 \%$ Tween-20 and $30 \mu \mathrm{M}$ steroid inducer Dex, deoxycorticosterone (Doc) or triamcinolone acetonide (TA) for $24 \mathrm{~h}$, then histochemically stained for GUS activity. (B) Leaf cuttings of the same leaf of 6-week-old plants (lines 65A, 121C) were induced in water supplemented with $0.1 \%$ Tween-20 and $10 \mu \mathrm{M}$ Dex or 10 $\mu \mathrm{M}$ TA for $24 \mathrm{~h}$, then histochemically stained for GUS activity. Leaves of pZmUbi::GUS were included as controls. Representative images are shown. (C) Leaves of 5- week-old plants (lines 65A top, 121C bottom) were induced by painting with water supplemented with $0.1 \%$ Tween-20 and $30 \mu \mathrm{M}$ steroid inducer: Dex, betamethasone (Bet), fludrocortisone acetate (Flu), prednisone (Pre) or prednisolone (Plo) for $24 \mathrm{~h}$, then histochemically stained for GUS activity. 

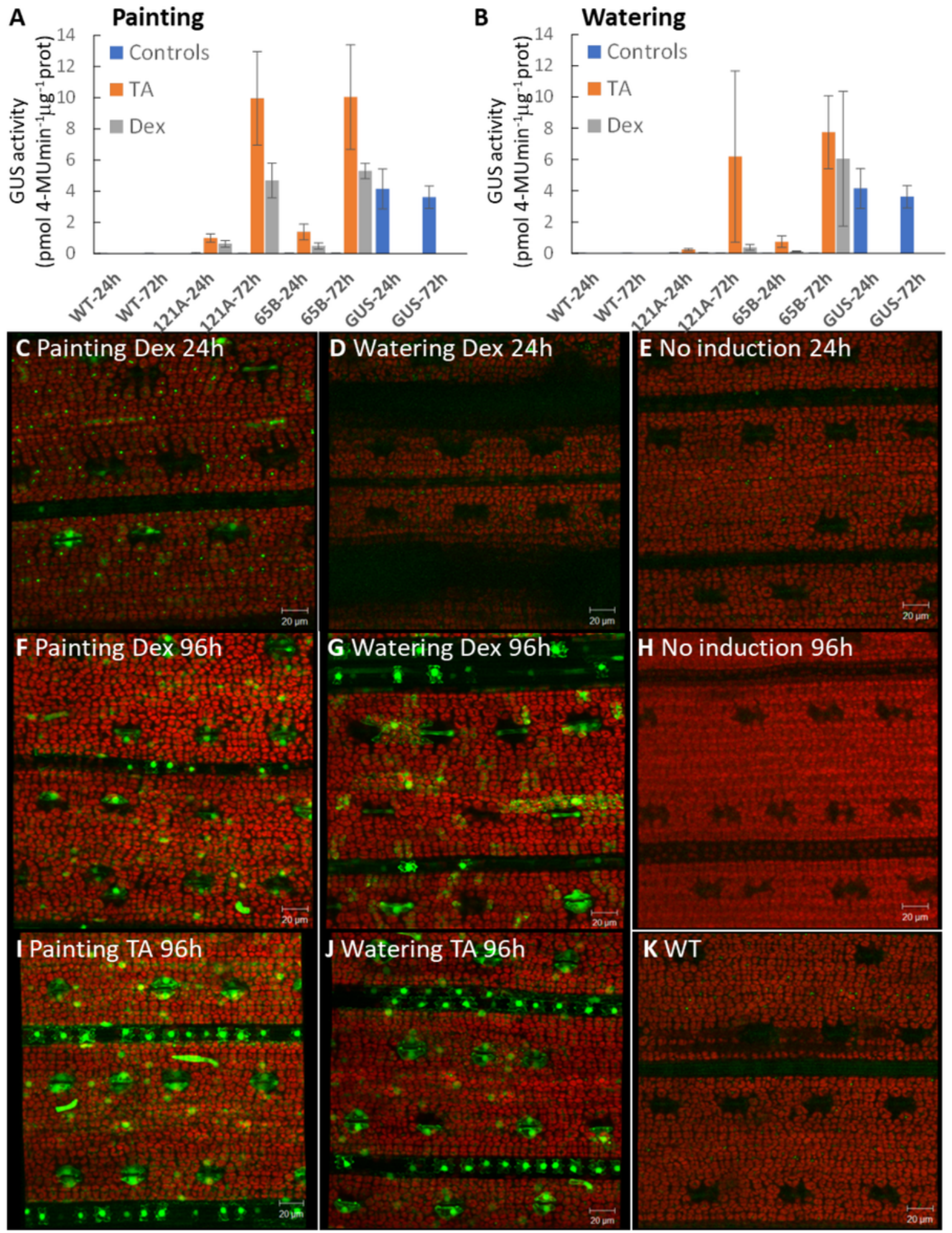

\section{Figure 5}

Expression characteristics of the p0p6/LhGR system induced in soil-grown plants by painting and watering Four-week-old rice plants grown in soil were induced either with TA or Dex by (A) painting with $10 \mu \mathrm{M}$ solution supplemented with $0.1 \%$ Tween-20 or (B) watering with $30 \mu \mathrm{M}$ solutions. The treatment was repeated twice at $0 \mathrm{~h}$ and $24 \mathrm{~h}$ later. Leaves were sampled and GUS activity determined $24 \mathrm{~h}$ and $72 \mathrm{~h}$ following first application of the inducer. WT and pZmUbi::GUS plants were used as controls. Three 
plants from segregating populations were used for each treatment. The error bars represent SD. (C-K) Four-week-old plants (line 121C) were induced as above except the treatment was repeated 48h after first induction. Leaves were imaged using a confocal laser scanning microscope at $24 \mathrm{~h}$ and $96 \mathrm{~h}$ postinduction, YFP signal in green, chlorophyll autofluorescence in red. Scale bar is $20 \mu \mathrm{m}$.

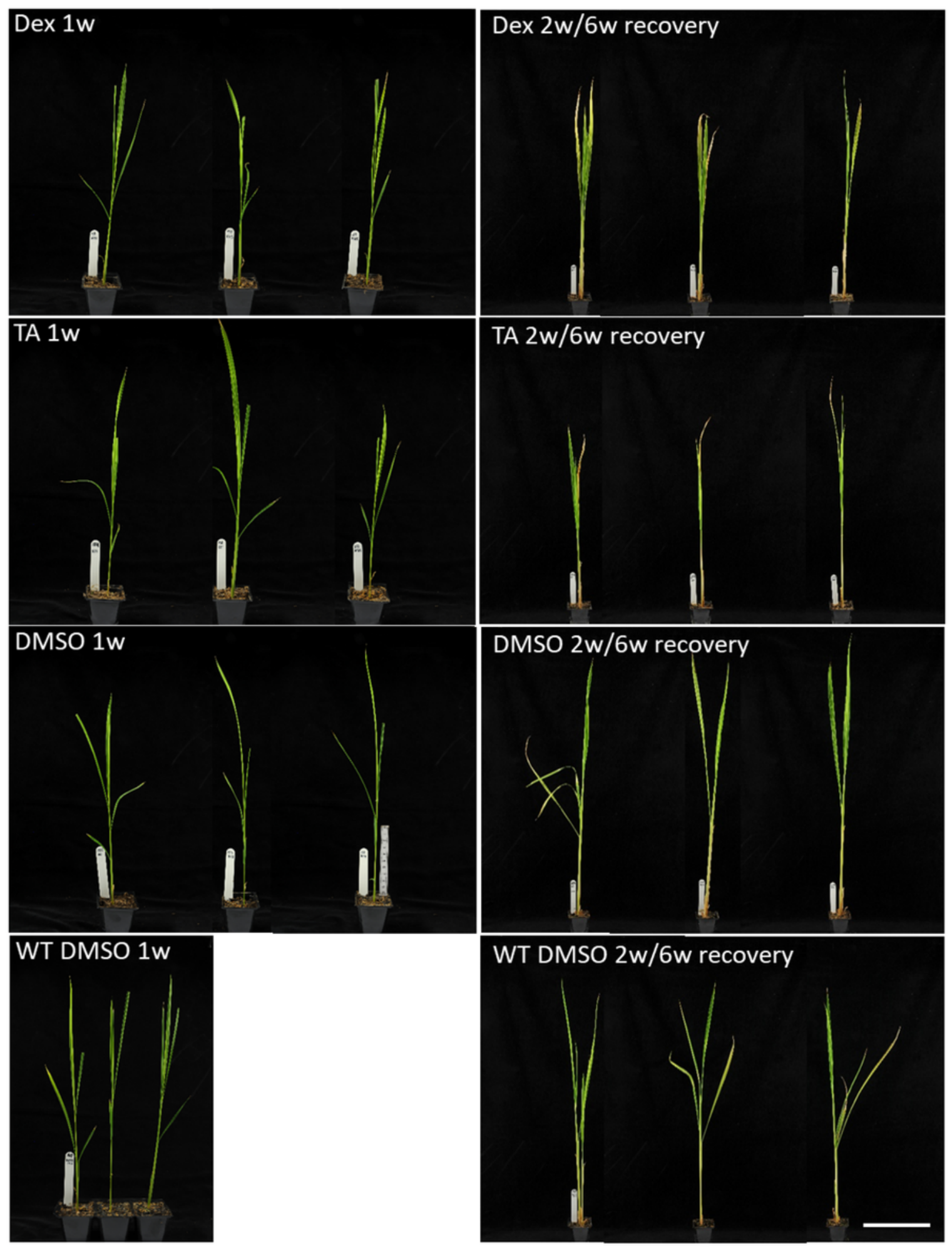

Figure 6 
Long-term effect of the p0p6/LhGR system induction on mature rice plants Four-week-old plants (65B and $121 \mathrm{~A})$ were watered with $30 \mu \mathrm{M}$ Dex or $30 \mu \mathrm{M}$ TA or a control solution containing DMSO $(600 \mathrm{ml}$ per a tray of 8 plants) at 2-day intervals for a week (left) or two weeks and let to recover for further 6 weeks (right). WT plants treated with the DMSO solution were used as controls. Scale bar represents $10 \mathrm{~cm}$. Representative images are shown.

\section{Supplementary Files}

This is a list of supplementary files associated with this preprint. Click to download.

- s1.png 